\title{
ETNOBOTÁNICA Y MORFO-ANATOMÍA COMPARADA DE TRES ESPECIES DE TAGETES QUE SE UTILIZAN EN Nicolás Romero, Estado de México
}

\author{
Fátima García-Sánchez ${ }^{1}$, Ma. Edith López-Villafrancoํ, Silvia Aguilar-Rodríguezz", 4 , \\ Abigall AGUILAR-CONTRERAS ${ }^{3}$ \\ ${ }^{1}$ Herbario IZTA, Facultad de Estudios Superiores Iztacala, \\ Universidad Nacional Autónoma de México, Tlalnepantla, México \\ ${ }^{2}$ Unidad de Morfología y Función, Facultad de Estudios Superiores Iztacala, \\ Universidad Nacional Autónoma de México, Tlalnepantla, México \\ ${ }^{3}$ Herbario IMSSM, Instituto Mexicano del Seguro Social, México, D.F. \\ ${ }^{4}$ Autor para la correspondencia: siagro@campus.iztacala.unam.mx
}

\begin{abstract}
Resumen: Tagetes lucida (pericón), T. lunulata (cinco llagas) y T. micrantha (anisillo), especies utilizadas como recurso terapéutico tradicional en el municipio Nicolás Romero, Estado de México, se estudiaron con la finalidad de aportar información a la farmacopea herbolaria de México. El trabajo etnobotánico se llevó a cabo por medio de entrevistas semiestructuradas a la población en general y para el estudio morfo-anatómico se recolectaron ejemplares en campo. Los tallos y hojas se cortaron a mano alzada y con un micrótomo de rotación y los cortes se tiñeron con safranina-verde rápido. Las especies se reconocieron para tratar dolor de estómago y diarrea, como tranquilizantes y para contrarrestar los cólicos menstruales. La manera frecuente de prepararlas es en infusión. El análisis morfológico mostró que los caracteres que más contribuyen para diferenciar entre especies son los relacionados con la arquitectura foliar. Anatómicamente las especies se distinguen por los tricomas, el arreglo y número de los cordones vasculares del mesofilo en la hoja y por el arreglo del córtex y del esclerénquima en el tallo. Se incluye una clave basada en caracteres morfo-anatómicos.
\end{abstract}

Palabras clave: anatomía vegetal, anisillo, Asteraceae, cinco llagas, herbolaria mexicana, pericón, plantas medicinales, Tagetes lucida, Tagetes lunulata, Tagetes micrantha.

\begin{abstract}
Tagetes lucida (pericón), T. lunulata (cinco llagas), and T. micrantha (anisillo), traditionally used as therapeutic resource in the locality of Nicolas Romero, Estado de México, were studied in order to provide information to the herbal pharmacopoeia of Mexico. Ethnobotanical work was conducted through semi-structured interviews to the general population and for morpho-anatomical research, field specimens were collected. The stems and leaves were sectioned by free hand and by using a rotary microtome and stained with safranin-fast green. The interviews showed that the species are used to treat stomach ache and diarrhea, as a relaxing agent, and to relieve menstrual cramps. The most common way to prepare them is as a tea. The morphological analysis showed that vegetative characters that provide more information to differentiate among species are related to the leaf architecture. Anatomically, the distinctive features are trichomes, distribution and number of vascular strands in the leaf mesophyll, and distribution of the cortex and sclerenchyma in the stem. This work also includes a key based on morphological and anatomical characters.
\end{abstract}

Key words: anisillo, Asteraceae, cinco llagas, medicinal plants, mexican herbal, pericón, plant anatomy, Tagetes lucida, Tagetes lunulata, Tagetes micrantha.

$\mathbf{E}^{1}$ género Tagetes L. cuenta con aproximadamente 50-55 especies ampliamente distribuidas en el territorio nacional, considerado posiblemente como el centro de origen y diversidad del género (Strother, 1977; Rzedowski, 2001; Villarreal, 2003; Villaseñor et al., 2005). Varias de sus especies se emplean como recurso terapéutico herbolario o como plantas ceremoniales y de ornato, entre otros (Caballero, 1990; Aguilar et al., 1994; Argueta-Villamar et al., 1994; Serrato-Cruz, 2003; Serrato-Cruz et al., 2008; Villavicencio-Nieto et al., 2010). Tagetes lucida y T. micrantha tienen importancia en la producción y uso de aceites esenciales con diversos fines (Bicchi et al., 1996; Ciccio, 2004). 
Numerosos trabajos hacen referencia a sus componentes fitoquímicos (Downum y Towers, 1983; Cáceres et al., 1992; Hernández et al., 2006) y otros a ensayos farmacológicos realizados en Tagetes spp. (Cambar et al., 2000). En el Estado de México, como en otras regiones del país, varias especies de Tagetes se llegan a ofrecer seccionadas para su venta en los tianguis regionales y mercados de zonas urbanas. Lo anterior crea la necesidad de contar con estudios que aporten información sobre sus usos, formas de preparación, administración y partes usadas, entre otras, así como para su determinación taxonómica. Esto último con la finalidad de contar con un control de calidad de las plantas que se comercializan. Algo sobre variabilidad morfológica intraespecífica en Tagetes se conoce por los trabajos de Serrato-Cruz y Barajas-Pérez (2006), mientras que caracteres anatómicos generales sólo se registran en Metcalfe y Chalk (1979) y Simon et al. (2002), quienes han referido la presencia de estructuras secretoras en la mayoría de las especies de este género. Con la finalidad de conocer aspectos sobre los usos y caracteres diagnósticos de Tagetes lucida Cav. (pericón), T. lunulata Ort. (cinco llagas) y T. micrantha Cav. (anisillo), para su inclusión en la Farmacopea Herbolaria de los Estados Unidos Mexicanos (CPFEUM, 2001), se llevó a cabo un estudio etnobotánico y la comparación morfo-anatómica del tallo y de la hoja de estas tres especies, procedentes del municipio de Nicolás Romero, Estado de México, México.

\section{Materiales y métodos}

Área de estudio. El municipio de Nicolás Romero, antiguamente llamado Azcapotzaltongo, palabra náhuatl que se traduce como "En los pequeños hormigueros", está formado por el conjunto montañoso Sierra de Monte Alto, y se ubica en la región noroeste del Estado de México a los 19³3' 50" N y $99^{\circ} 15^{\prime}$ '53" O, a una altitud de 2,370 m. Su clima es templado subhúmedo con lluvias en verano y tiene una temperatura media de $16{ }^{\circ} \mathrm{C}$ (García, 1973). La vegetación corresponde a un bosque de pino-encino según la clasificación de Rzedowski (1978). Los árboles que caracterizan esta comunidad vegetal pertenecen a los géneros Abies, Pinus y Quercus; en el estrato arbustivo se encuentran Gaultheria, Juniperus, Montanoa y en el herbáceo Erigeron, Iresine, Salvia, Senecio y Stevia. En los huertos familiares predominan especies de las familias Asteraceae, Lamiaceae, Rosaceae, Rutaceae y Verbenaceae, Este municipio está habitado por hñahñu, mazahuas y mestizos (Zolla y Zolla-Márquez, 2004).

Etnobotánica. La información se recabo siguiendo las recomendaciones de Martínez-Alfaro (1976), Hernández-X (1979) y Cotton (1996). Para reconocer las especies se hicieron recorridos en campo conjuntamente con los informantes. El conocimiento que se tiene en el área de trabajo sobre el uso de las tres especies de Tagetes, se adquirió por medio de preguntas abiertas. Los entrevistados se seleccio- naron al azar en las localidades los Jarros, Benito Juárez Barrón y el mercado municipal de San Pedro. Para la recolección y el trabajo de herbario se siguió lo propuesto por Lot y Chiang (1986). Los ejemplares se incluyeron en la colección etnobotánica del Herbario (IZTA) de la Facultad de Estudios Superiores Iztacala y en el Herbario (IMSSM) del Instituto Mexicano del Seguro Social. Los números de registro son: Tagetes lucida 1798-IZTA, 15805-IMSS; T. lunulata 1800-IZTA, 15806-IMSS; T. micrantha 1799-IZTA, 15807-IMSS.

Morfología. Tomando como base la descripción de Rzedowski (2001) se examinaron algunos caracteres morfológicos de individuos recolectados en campo. Debido a que los entrevistados reconocen a las especies de Tagetes frecuentemente por la flor, se proporciona información sobre sus caracteres. También se revisaron datos sobre altitud, hábito, y tamaño del individuo para cada especie.

Anatomía. Se recolectaron cuatro individuos de cada especie, inmediatamente después se fijaron tallos completos y hojas en formol $10 \mathrm{ml}$, ácido acético glacial $5 \mathrm{ml}$, agua destilada $35 \mathrm{ml}$ y alcohol al $96 \% 50 \mathrm{ml}$. Se realizaron cortes a mano alzada en sección transversal de la parte basal, media y superior de los tallos, así como en la parte media de la hoja de las tres especies y de los segmentos de Tagetes lunulata y $T$. micrantha. Las secciones se tiñeron con azul de toluidina, cristal violeta y safranina para su posterior montaje en gelatina glicerinada (Aguilar-Rodríguez, 1998). Otras muestras de tallo y hoja se deshidrataron en una serie gradual de alcohol etílico, posteriormente se trataron con xilol y se incluyeron en parafina histológica a $58-60{ }^{\circ} \mathrm{C}$ (Ruzin, 1999). Con un micrótomo de rotación se obtuvieron secciones transversales de $17 \mu \mathrm{m}$ de grosor. La tinción de los cortes se hizo con safranina “O”-verde rápido FCF y se montaron en resina sintética. La epidermis se obtuvo mediante la técnica de raspado (Aguilar-Rodríguez, 1998) e impresión de negativos (Sandoval-Z, 2005). Las descripciones anatómicas se realizaron siguiendo los términos empleados por Metcalfe y Chalk (1979) y Fahn (1985). Para los caracteres del xilema secundario en los tallos, se siguió lo propuesto por la Asociación Internacional de Anatomistas de la Madera (IAWA Committee, 1989). Se describió el tipo de porosidad, agrupación y diámetro del lumen de los vasos; de este último se proporciona la media, la desviación estándar y los valores máximos y mínimos. Para otros caracteres de hoja y/o tallo como el tamaño de células epidérmicas, grosor de la cutícula, tamaño de los tricomas, tamaño de células del mesofilo y de los paquetes de esclerénquima, se muestra el valor máximo y mínimo. De cada muestra se tomaron 25 mediciones; éstas, así como las observaciones y digitalización de imágenes, se realizaron con el analizador de imágenes NIS- elements BR 2.33 (Nikon Corporation, 1991-2006). Los dibujos de tallo y hoja se elaboraron em- 
pleando una cámara clara a 20X en un microscopio marca Nikon Labophot-2.

\section{Resultados}

Etnobotánica. Para conocer los uso medicinales que los habitantes de Nicolás Romero les proporcionan a las tres especies de Tagetes, se entrevistaron a 48 habitantes de la zona de estudio: amas de casa (73\%), comerciantes $(17 \%) \mathrm{y}$ agricultores (10\%). El $85 \%$ fueron mujeres y el 15\% hombres. Todas las personas entrevistadas reconocieron a las especies; $79 \%$ de los pobladores reconoció a las tres especies, $17 \%$ sólo dos (T. lucida y/o T. lunulata y/o T. micrantha) y $4 \%$ sólo a una de las tres. Los caracteres que se hicieron evidentes para identificar a las tres especies de Tagetes se basaron principalmente en la flor (59\%) y en el aroma (31\%); otros caracteres fueron la forma de la hoja y el sabor o el color (10\%). Para el caso de T. micrantha, fue el olor a anís, mientras que para el pericón (T. lucida) y el cempasúchil (T. lunulata) fue la flor, pues ésta es llamativa en ambas especies. La categoría de edad mejor representada para las mujeres fue de 35 a 65 años, mientras que para los hombres fue de 48 a 67 años. Así tenemos que en Nicolás Romero $100 \%$ de las personas entrevistadas dijo conocer a T. lucida como "pericón"; sin embargo, a T. lunulata se le asignan diferentes nombres populares, el $41 \%$ dijo conocerla como "cempasúchil cimarrón”, $37 \%$ como "cempasúchil” y 22\% como "cinco llagas". El 64\% de la población denomina a T. micrantha con el nombre de "anisillo", 20\% como "anís de campo" y 16\% como "yerbanis". Por otro lado, consideran al pericón y al anisillo plantas calientes ya que suelen "atemperar" el estómago, mientras que el "cinco llagas" tiene la dualidad de ser fría o caliente. Dentro de las nosologías más frecuentes que padecen los habitantes en el área de estudio, están en primer lugar los malestares estomacales, que indican los pobladores se manifiestan durante todo el

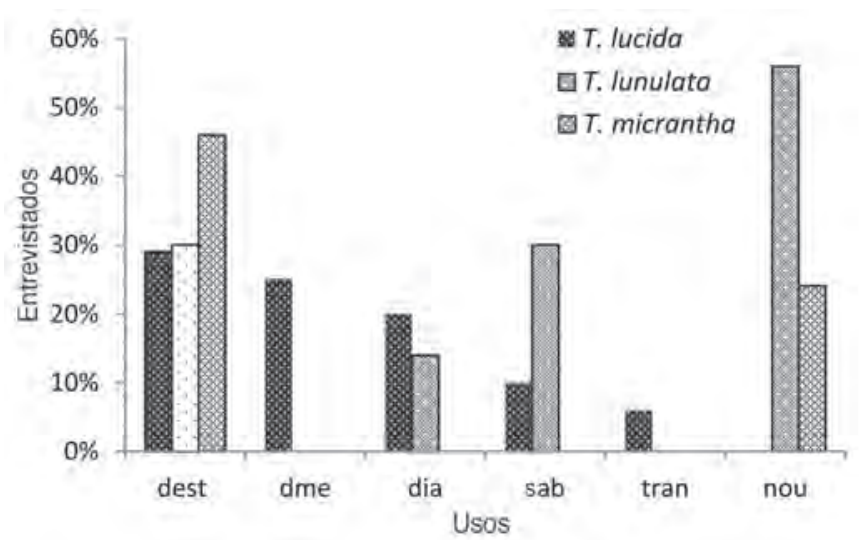

Figura 1. Usos que dan los habitantes de Nicolás Romero, Estado de México a las tres especies de Tagetes expresados en porcentajes. dest $=$ dolor de estómago, dia $=$ diarrea, dme $=$ dolor menstrual, tran $=$ tranquilizante, $\mathrm{sab}=$ saborizante, nou $=$ no la utiliza. año y refieren que existe una temporada a la que reconocen como "la canícula", definida como la época más caliente del año, en la que estos padecimientos se intensifican. Se registró que las tres especies se emplean principalmente en el tratamiento de enfermedades gastrointestinales: dolor de estómago, controlar la diarrea y el empacho; así como para el dolor menstrual y como tranquilizante. También se emplean para saborizar atoles y dar color y aroma a los elotes y chayotes hervidos (Figura 1). La especie que registró más usos fue T. lucida (pericón), seguida de T. lunulata (cempasúchil) y T. micrantha (anisillo). Dentro de las estrategias que tienen los habitantes entrevistados de Nicolás Romero para prevenir y curar las enfermedades, está el conocimiento médico tradicional popular que posee cada familia. Éste generalmente consiste en tomar un té de alguna planta para que les alivie el dolor y si no se calma, como una opción secundaria recurren a la medicina alópata. Las tres especies de Tagetes se usan sólo cuando se encuentran disponibles, el resto del año se consume ajenjo (Artemisia absinthium), manzanilla (Matricaria recutita) y hierbabuena (Mentha sp.). Con respecto al modo de preparación, las tres especies de Tagetes se utilizan de manera similar, esto es, en infusión con o sin azúcar, por ejemplo: “... se consigue un manojo de pericón, se pone a hervir agua y cuando esté hirviendo se le agrega, pero sin raíz. Se le puede poner azúcar o no y se toma calientito...". En general, solo se utiliza la parte aérea de las plantas. En el caso de que se empleen en forma de condimento para dar sabor "... cuando hagas atole, se lo pones cuando le vayas a echar la harina para que te sepa a anís....". En cualquiera de las formas de preparación no

Cuadro 1. Datos de altitud y algunas características morfológicas de tres especies de Tagetes (Información modificada de Rzedowski, 2001).

\begin{tabular}{|c|c|c|c|}
\hline & T. lucida & T. lunulata & T. micrantha \\
\hline Hábito & perenne & anual & anual \\
\hline Altitud & $2,500-2,900$ & $2,500-3,000$ & $2,500-3,000$ \\
\hline Tamaño & hasta $80 \mathrm{~cm}$ & hasta $80 \mathrm{~cm}$ & hasta $40 \mathrm{~cm}$ \\
\hline $\begin{array}{l}\text { Forma de } \\
\text { la hoja }\end{array}$ & $\begin{array}{l}\text { entera, linear } \\
\text { a oblonga } \\
\text { elíptica u } \\
\text { oblanceolada }\end{array}$ & $\begin{array}{l}\text { pinnatisecta: } \\
\text { segmentos de } \\
9 \text { a 23, lineares } \\
\text { a lanceolados }\end{array}$ & $\begin{array}{l}\text { pinnatisecta: } \\
\text { segmentos } \\
\text { de } 3 \text { a } 9\end{array}$ \\
\hline $\begin{array}{l}\text { Margen de } \\
\text { la hoja }\end{array}$ & aserrado & $\begin{array}{l}\text { segmentos } \\
\text { profundamente } \\
\text { aserrados }\end{array}$ & $\begin{array}{l}\text { segmentos } \\
\text { enteros }\end{array}$ \\
\hline Flores liguladas & $\begin{array}{l}\text { amarillas de } \\
3 \text { a } 4\end{array}$ & $\begin{array}{l}\text { amarillas a } \\
\text { naranjas } 5 \text { con } \\
\text { una marca de } \\
\text { "V" o "W" en } \\
\text { la base }\end{array}$ & $\begin{array}{l}\text { blancas de } \\
0 \text { a } 3\end{array}$ \\
\hline Flores del disco & 5 a 8 & 20 a 40 & 4 a 9 \\
\hline Fruto & $\begin{array}{l}\text { aquenio linear- } \\
\text { claviforme }\end{array}$ & aquenio linear & aquenio linear \\
\hline
\end{tabular}




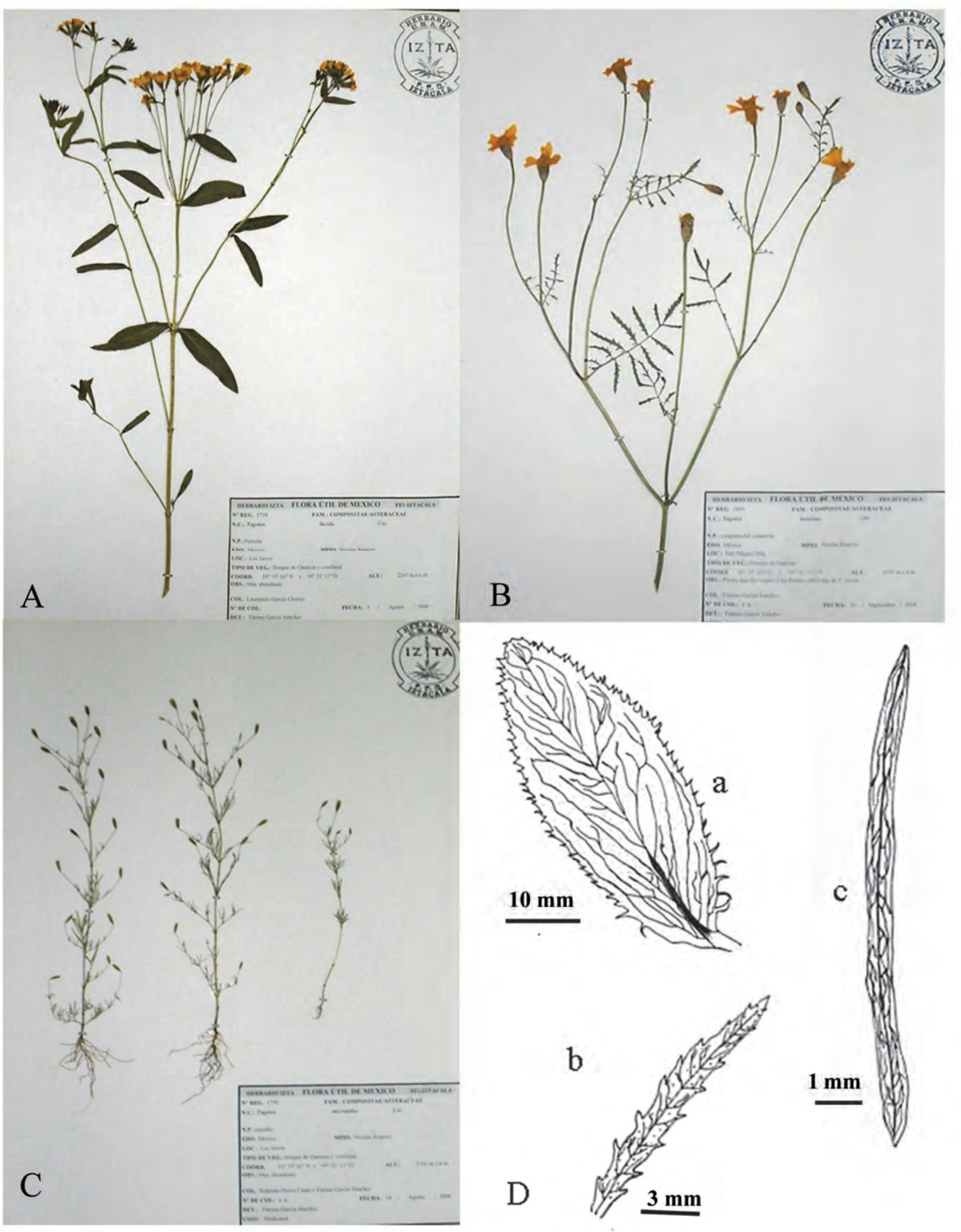

Figura 2. Morfología de tres especies de Tagetes. A. T. lucida. B. T. lunulata. C. T. micrantha. D. Detalle de hojas. a = hoja de T. lucida. $\mathrm{b}=$ segmento de $T$. lunulata $\mathrm{c}=$ segmento de $T$. micrantha. 
se mencionó interacciones o mezclas con otras especies de plantas. Haciendo referencia a la época de colecta, $58 \%$ de los entrevistados comenta que las tres especies de Tagetes se encuentran disponibles y frescas, principalmente en estación de lluvias. También mencionaron que el pericón (T. lucida) puede crecer todo el año, pero que es muy difícil conseguirlo. Asimismo, 65\% de los pobladores mencionó que obtenía las plantas del monte, $24 \%$ de la orilla de los ríos y un $11 \%$ compra pericón y en ocasiones anisillo en los mercados. Ninguna de las plantas se almacena, pues son especies de recolecta que se emplean en fresco. De acuerdo a las respuestas de los agricultores, ninguna de las tres especies tienen algún tipo de manejo para su cultivo y los comerciantes indican que sólo el pericón (T. lucida) y el anís (T. micrantha) se recolectan en campo para su venta en el mercado local.

Morfología. En el cuadro 1 y figura 2 se muestran algunos aspectos morfológicos por los que se pueden reconocer las tres especies de Tagetes. Plantas herbáceas anuales o perennes, aromáticas. Sus hojas y brácteas involucrales con glándulas oleíferas translúcidas puntiformes o lineares; involucro cilíndrico, fusiforme o angostamente campanulado; hojas enteras a pinnatisectas, con margen entero a aserrado, flores liguladas dispuestas en la periferia de las cabezuelas de color blanco, amarillo o naranja; aquenios linear a linear- claviformes.

Anatomía. Lámina foliar. En vista superficial, células epidérmicas con paredes anticlinales sinuosas, gruesas en $\mathrm{Ta}$ getes lucida; estomas anomocíticos en la superficie abaxial (Figura 3A). En sección transversal de ambas superficies, cutícula lisa con 2-10 $\mu \mathrm{m}$ de espesor; epidermis simple, células epidérmicas de 5-64 $\mu \mathrm{m} \times 14-64 \mu \mathrm{m}$; mesofilo bifacial en $T$. lucida y $T$. lunulata (Figura 3B, C) y equibilateral en T. micrantha (Figura 3H, I). En las especies con mesofilo bifacial, el parénquima en empalizada formado por una capa de células de 32-76 $\mu \mathrm{m} \times 8-6 \mu \mathrm{m}$, ocupando el 24-30\% del mesofilo, el parénquima esponjoso de 4 a 5 capas, ocupando el 70-76\% del mesofilo, el mesofilo equibilateral formado por una capa de células en cada una de las superficies de la hoja, de 42-74 $\mu \mathrm{m} \times 2-17 \mu \mathrm{m}$; cavidades secretoras de origen esquizógeno en las tres especies (Figura 3L) y numerosos cordones vasculares de tipo colateral con una vaina de células parenquimatosas.

Vena media: Cutícula lisa con 4-13 $\mu \mathrm{m}$ de espesor, tricomas uniseriados de paredes gruesas en Tagetes lucida y T. lunula$t a$, en la primera especie los tricomas con una célula apical aguda, más abundantes en la superficie abaxial (Figura 3D), mientras que en $T$. lunulata estos tricomas en mayor número en la superficie adaxial; T. micrantha sin tricomas. En sección transversal, epidermis simple con células de 15-35 $\mu \mathrm{m} \times 12$ $41 \mu \mathrm{m}$ en las tres especies. Por debajo de la epidermis de ambas superficies de 1 a 4 capas de parénquima con diferentes grados de engrosamiento, desarrollando colénquima o células esclerosadas (Figuras 3E-G, J). Con dos cordones vasculares en $T$. lucida, uno más desarrollado que el otro, el mayor con más de 20 vasos xilemáticos organizados radialmente, cada uno con fibras extendiéndose a manera de arco en ambos polos, rodeados por una vaina de esclereidas (Figura 3E). Cinco cordones vasculares en $T$. lunulata, los de los extremos más pequeños y los tres centrales de mayor tamaño (Figura 3F); en los segmentos un sólo cordón vascular con15-20 vasos xilemáticos, organizados radialmente, la vaina formada por un estrato de células de parénquima con paredes delgadas (Figura 3G). Tres cordones vasculares en T. micrantha (Figura 3J), el central de mayor tamaño; en los segmentos un sólo cordón vascular con xilema poco desarrollado, con 6-10 vasos xilemáticos, rodeado por una vaina con 1-2 estratos de células parenquimáticas, por dentro de esta vaina un grupo de fibras asociadas al floema (Figura 3K).

El tallo, en sección transversal, de forma circular sinuosa en la base y en las partes más jóvenes del eje de forma cuadrangular (Figura 4A-C); cutícula, con un grosor de 4-9 $\mu \mathrm{m}$; tricomas simples, uniseriados de 4 a 6 células de paredes gruesas y escasos en Tagetes lucida (Figura 5A); tricomas glandulares con un pedicelo unicelular de 34-46 $\mu \mathrm{m}$ de largo y una célula apical redonda, translucida, de $40-70 \mu \mathrm{m}$ de diámetro en T. micrantha (Figura 5B) y tricomas ausentes en T. lunulata. Epidermis simple, con células de 7-24 $\mu \mathrm{m}$ $\times 20-53 \mu \mathrm{m}$ en T. lucida y T. micrantha y de 10-26 $\mu \mathrm{m} \times$ 20-66 $\mu \mathrm{m}$ en $T$. lunulata, esta especie ocasionalmente con las paredes de las células epidérmicas engrosadas y una peridermis incipiente (Figura 5C). El córtex formado por colénquima, parénquima, esclerénquima y canales secretores de origen esquizógeno; el colénquima angular de 4-5 capas, mejor desarrollado en los ángulos del tallo (Figuras 4A-C, $5 \mathrm{~F}$ ), el parénquima con grandes espacios intercelulares formando un aerénquima en $T$. lucida y T. micrantha (Figuras $4 \mathrm{~A}, \mathrm{C}, 5 \mathrm{E}$ ), pero en la parte media y superior del eje escasos o ausentes (Figura 4A, C), mientras que en T. lunulata siempre compacto (Figuras 4B, 5C). Por debajo con paquetes de esclerénquima organizados tangencialmente, formados por 4 a 7 hileras de células. En T. lunulata y T. micrantha los paquetes de esclerénquima generalmente separados por tejido parenquimático (Figura 4B, C); en T. lucida, más grandes que en las otras dos especies, separados por células esclerosadas (Figura 5E).

El xilema secundario con espesor de 449-785 $\mu \mathrm{m}$ en $\mathrm{Ta}$ getes lucida y 640-1,120 $\mu \mathrm{m}$ en T. lunulata, escaso (40-224 $\mu \mathrm{m})$ pero mayor acumulación en las partes sinuosas en $T$. micrantha. La porosidad difusa, diámetro tangencial del lumen de los vasos de $15 \pm 2.9 \mu \mathrm{m}(8-22 \mu \mathrm{m})$ en T. micrantha hasta $28.3 \pm 4.9 \mu \mathrm{m}(25-40 \mu \mathrm{m})$ en $T$. lunulata, vasos solitarios o agrupados en cadenas radiales de 3 a 7 (Figura 5D). Xilema primario en las zonas más jóvenes del eje en haces vasculares de tipo colateral. Médula parenquimatosa con forma de estrella, diámetro promedio de $1.72 \mathrm{~mm}$ en $T$. lucida y $1.34 \mathrm{~mm}$ en $T$. lunulata hasta $0.53 \mathrm{~mm}$ en $T$. micrantha (Figuras 4A-C). 


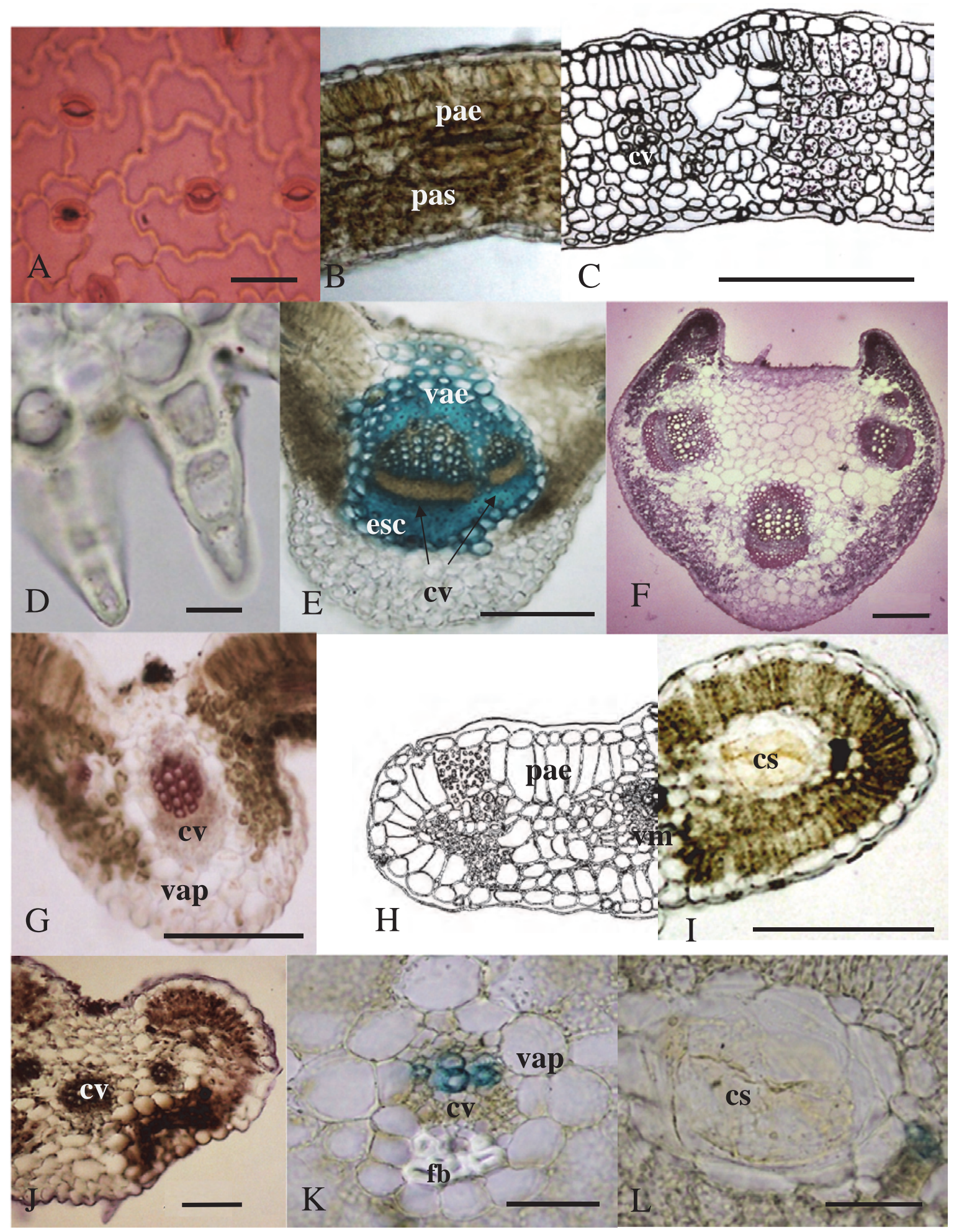

Figura 3. Hojas en vista superficial y transversal de Tagetes. A-E. T. lucida. A. Estomas anisocíticos. B, C. Mesofilo bifacial. D. Tricomas simples en la superficie abaxial de la vena media. E. Vena media con dos cordones vasculares rodeados de una vaina de esclerénquima. F,G. T. lunulata. F. Vena media mostrando 5 cordones vasculares. G. Vena media del segmento mostrando un cordón vascular. H-L. T. micrantha. H, I. Vista transversal del segmento. J. Vena media mostrando 3 cordones vasculares. K. Vena media del segmento. L. Canal secretor en el mesofilo. $\mathrm{cs}=$ canal secretor, $\mathrm{cv}=$ cordón vascular, esc $=$ esclerénquima, $\mathrm{fb}=$ fibras asociadas al cordón vascular, pae $=$ parénquima en empalizada, pas = parénquima esponjoso, vae = vaina de células esclerosadas, vap = vaina de parénquima, vm $=$ vena media. (Escalas: A, D, K, L $=50 \mu \mathrm{m} ; \mathrm{B}, \mathrm{C}, \mathrm{E}-\mathrm{I}=200 \mu \mathrm{m} ; \mathrm{J}=100 \mu \mathrm{m}$ ). 

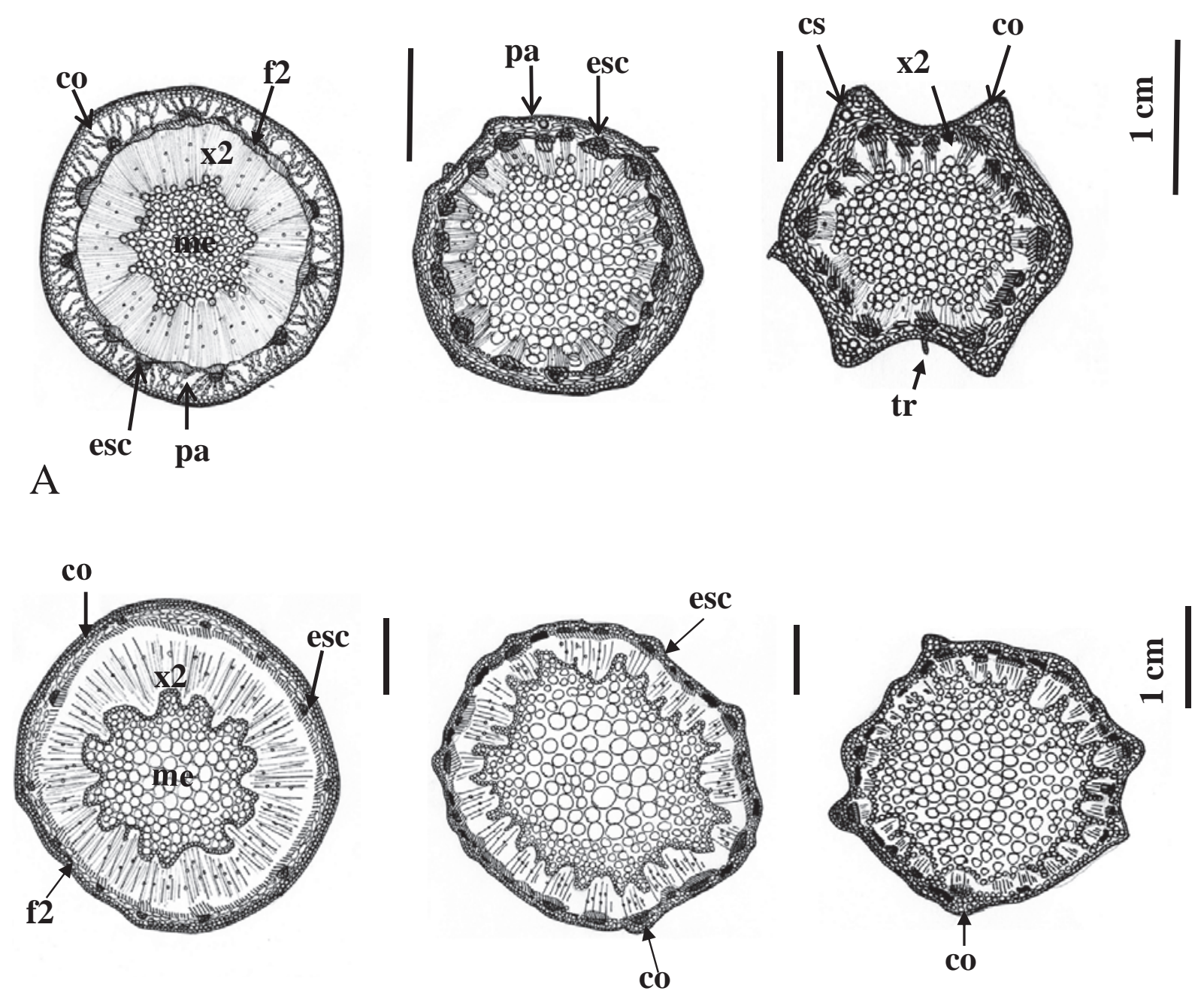

B
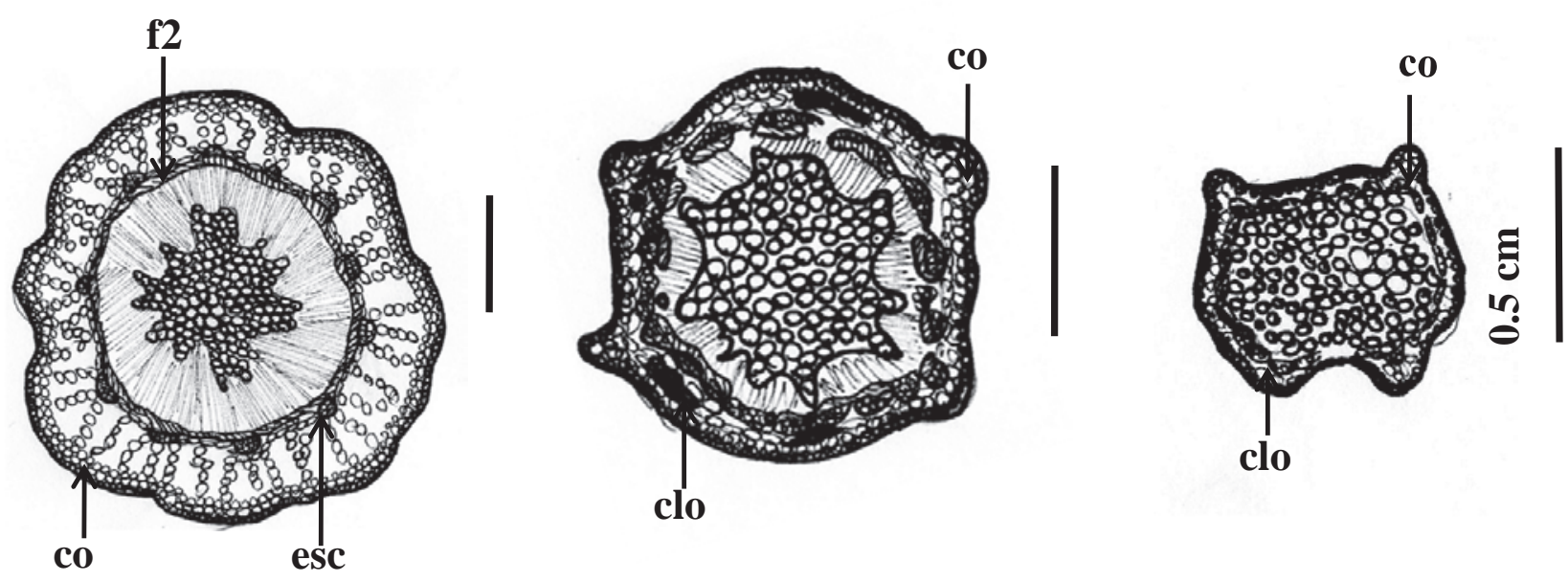

C

Figura 4. Vistas trasversales de tallo basal, medio y superior de Tagetes. A. T. lucida. B. T. lunulata. C. T. micrantha. clo = clorénquima, $\mathrm{co}=$ colénquima, esc $=$ esclerénquima, $\mathrm{f} 2=$ floema secundario, $\mathrm{pa}=$ parénquima, $\mathrm{me}=$ médula, $\mathrm{tr}=$ tricoma, $\mathrm{x} 2=\mathrm{xilema}$ secundario. 


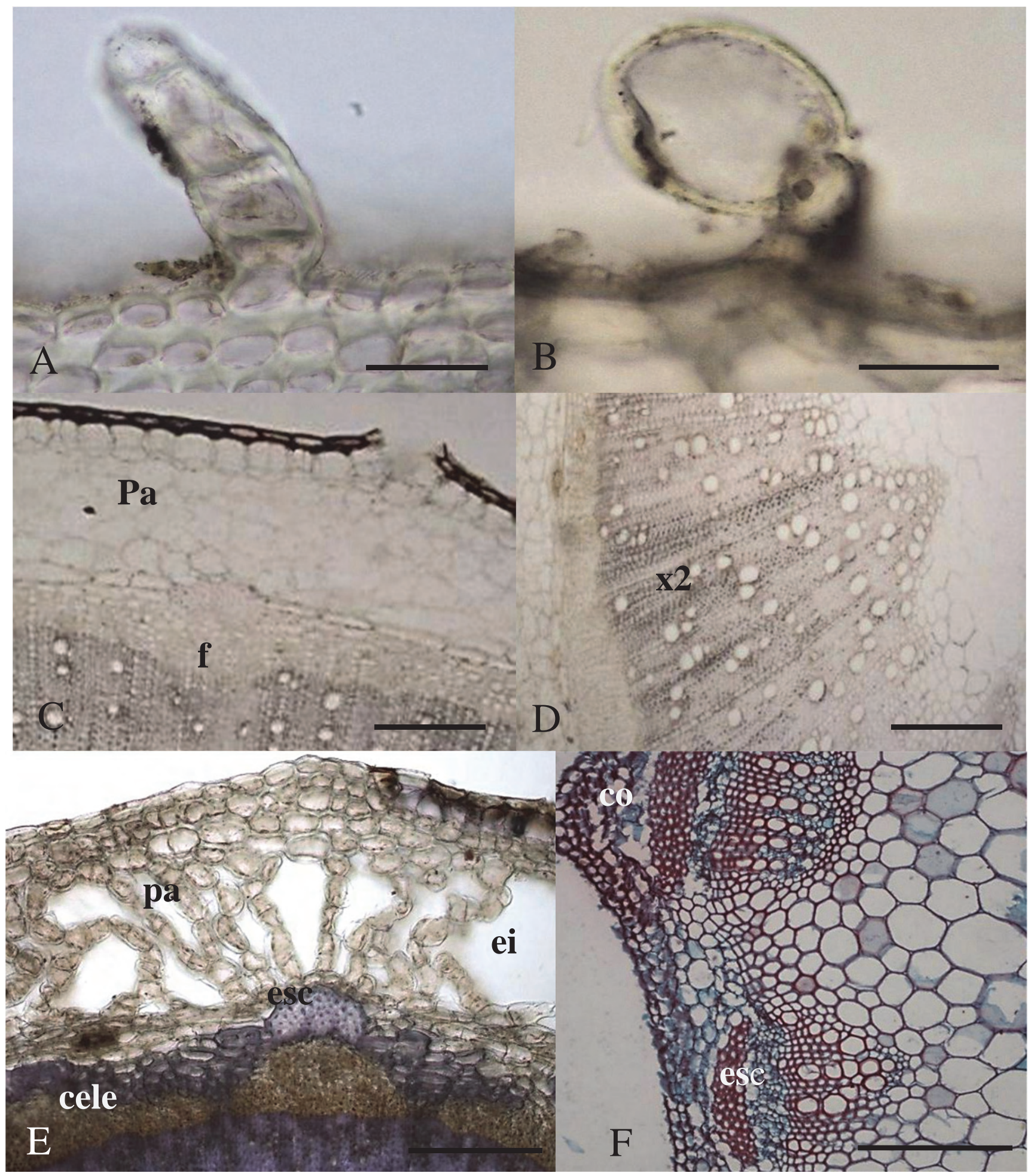

Figura 5. Vistas trasversales de tallo de Tagetes. A. Tricoma simple con 3 células en T. lucida. B. Tricoma glandular en T. micrantha. C,D. T. lunulata. C. Córtex. D. xilema secundario mostrando vasos solitarios y agrupados en cadenas en tallo basal. E. Aerénquima del córtex en tallo basal de T. lucida. F. Arreglo de los haces vasculares en el tallo superior de T. lunulata. cele = células esclerosadas, co = colénquima, ei $=$ espacio intercelular, esc $=$ esclerénquima, $\mathrm{f}=$ floema, $\mathrm{pa}=$ parénquima, $\mathrm{x} 2=$ xilema secundario. $($ Escalas: $\mathrm{A}, \mathrm{B}=50 \mu \mathrm{m}$;

$$
\mathrm{C}-\mathrm{F}=200 \mu \mathrm{m}) \text {. }
$$


Clave para la identificación de las especies con base en caracteres morfo-anatómicos.

1. Hojas con lámina entera, vena media con dos cordones vasculares; vaina del haz formada por células esclerosadas T. lucida

1. Hojas con lámina pinnatisecta, vena media con más de dos cordones vasculares; en los segmentos con sólo un cordón vascular, vaina del haz formada por células de parénquima.

2. Segmento de la lámina foliar con margen aserrado, mesofilo bifacial y cinco cordones vasculares

T. lunulata

2. Segmento de la lámina con margen entero, mesofilo equibilateral y tres cordones vasculares

T. micrantha

\section{Discusión}

Etnobotánica. Desde la época prehispánica se han utilizado diferentes especies de Tagetes como recurso terapéutico y las comunidades indígenas y mestizas del país siguen empleándolas para contrarrestar principalmente diversos trastornos digestivos (de la Cruz y Badiano, 1991; Aguilar et al., 1994), lo que las convierte en un recurso curativo importante. Las entrevistas realizadas en Nicolás Romero permitieron constatar que T. lucida, T. lunulata y T. micrantha se utilizan de manera regular como plantas medicinales y saborizantes. Las entrevistas, en primera instancia, se hicieron a las mujeres, pues ellas tienen una participación importante dentro del saber médico tradicional, en otras regiones del país y del mundo (Campos-Navarro, 1997; Zolla et al., 1998; Howard, 2003). El conocimiento y manejo de las plantas por parte de las mujeres, contribuye a la conservación y al uso sustentable de los recursos vegetales en sus comunidades (Howard, 2003). El trabajo en campo permitió observar que las plantas recolectadas por los habitantes de Nicolás Romero para uso personal o comercialización, crecen silvestres a orillas de caminos, o son toleradas en los sembradíos de maíz, haba o frijol. En otras regiones del país diversas especies de Asteraceae, Fabaceae y Poaceae, entre otras, son toleradas entre las milpas con fines medicinales (Lira et al., 2009). De acuerdo a los informantes, las especies estudiadas son abundantes durante época de lluvias; sin embargo, T. lucida (pericón) se encuentra disponible todo el año, aunque su colecta se restringe al fondo de las barrancas, donde la humedad es constante. Al respecto, SerratoCruz (2003) menciona que es mejor recolectar a Tagetes, en época de lluvias, pues cuando las plantas se encuentran en floración conservan mejor sus principios activos. Tagetes lucida resultó ser la más conocida en Nicolás Romero, la cual se denomina popularmente "pericón", seguida de $T$. micrantha, que por su aroma y sabor lo nombran "anisillo" y T. lunulata, a la cual llaman "cempasúchil cimarrón"; estas especies también se les reconoce con diferentes nombres populares en otras partes de la República (Aguilar et al., 1994). Desde el punto de vista médico popular se tomó en cuenta el síndrome de la dualidad frío/calor de las plantas medicinales, en general este concepto está ampliamente difundido en América (Nates-Cruz et al., 1996). Para el caso de las tres especies estudiadas, los habitantes indicaron que T. lucida y T. micrantha son plantas de calidad caliente ya que suelen "atemperar" el estómago, mientras que para $T$. lunulata, la opinión se dividió y algunas mencionaron que es de naturaleza fría y otras que es caliente. Como lo indica Foster (1988), comúnmente las personas utilizan plantas calientes para contrarrestar enfermedades frías, por ejemplo, las respiratorias (catarros y gripa), además de algunos trastornos digestivos (disentería blanca, flatulencia y cólicos menstruales). Asimismo, se toma en consideración que las plantas amargas y muy aromáticas, tienden a ser reconocidas dentro de la categoría de plantas calientes (Foster, 1988), como sucede en las tres especies estudiadas, pues $T$. lucida y T. micrantha se apreciaron por el olor fuertemente anisado, mientras que T. lunulata se percibió con olor fuerte y sabor amargo.

Los principales usos que se les dieron a las tres especies de Tagetes están relacionados con las enfermedades gastrointestinales, resultados que concuerdan con lo reportado por Osuna-Torres et al. (2005), quienes mencionan que algunas especies de este género se utilizan actualmente para el tratamiento de diarrea y disentería y se ingieren solas o mezcladas con hierbabuena (Mentha sp.) o guayaba (Psidium guajava). Por su parte, Serrato-Cruz (2003) refiere que debido a la presencia de alcaloides, ácido tánico y tiofenos, las especies de Tagetes se utilizan para combatir diversos microorganismos patógenos que suelen estar relacionados con el dolor de estómago, además de servir como estimulantes estomacales. Recientemente, Alonso-Castro et al. (2011) reportan 300 especies que se emplean para el tratamiento de cáncer y listan a T. lucida como un recurso médico popular en el caso de cáncer gástrico. Por otro lado, T. lucida y T. micrantha también se emplean como saborizantes por su olor a anís y sabor dulce, usos que se registran desde épocas prehispánicas (de la Cruz y Badiano, 1991).

Morfología. Las tres especies se diferencian principalmente por la morfología de sus hojas, su aroma y el involucro floral. Tagetes micrantha es la especie más pequeña. En varios estudios se hace referencia al ambiente como un factor que influye en la talla de las especies (Fahn, 1985 Aguilar-Rodríguez et al., 2006; Moles et al., 2009). En el sitio de estudio T. micrantha se desarrolla en áreas abiertas, a la orilla de caminos y en suelos pobres, compactos y con poca humedad, probablemente esto influye en el desarrollo limitado de sus tallos. Las otras dos especies crecen (incluso juntas) en sitios más húmedos o asociadas con cultivos, lo que probablemente favorece la formación de tallos más robustos o de mayor tamaño. Otra diferencia es la morfología 
de sus hojas. Tagetes lunulata y T. micrantha tienen hojas pinnatisectas, pero en $T$. micrantha son pequeñas y gruesas. Esta última característica se refleja claramente en sus tejidos, pues es la única especie que mostró un mesofilo equibilateral en sus segmentos foliares. La literatura refiere que $T$. lunulata y T. micrantha presentan hojas pinnadas (Rzedowski, 2001, Villarreal, 2003). Sin embargo, el análisis microscópico de la vena media, muestra que en ambas especies se extienden lateralmente reminiscencias de la lámina foliar. Con base en esta observación, en el presente trabajo se adopta el término "pinnatisecta", con base a la descripción de Font Quer (1982).

Anatomía. Las Asteraceae poseen una estructura anatómica muy diversa que puede variar dependiendo del hábitat y de las especializaciones ecológicas de cada especie (Metcalfe y Chalk, 1979). A pesar de esto parece que la formación de canales secretores de origen esquizógeno es común en la familia, carácter que se presentó en el tallo y en las hojas de las tres especies de Tagetes. Dichas estructuras son descritas por Simon et al. (2002) para T. minuta como cavidades secretoras más o menos esféricas que presentan un epitelio multiseriado, similar a lo observado en las especies estudiadas, aunque aquí se reconocen como canales secretores. En estos canales también es común la presencia de compuestos con apariencia aceitosa que en T. lucida se han identificado principalmente como estragol (Bicchi et al., 1996; Ciccio, 2004) y en $T$. erecta son piperitona, piperitenona, indol y $\beta$-ocimene (Russin et al., 1988; Ogunwande y Olawore, 2006). En general, los aceites esenciales de Tagetes podrían funcionar como un mecanismo de protección contra herbívoros y como repelente de insectos, además de tener aplicaciones agroquímicas, farmacológicas y aromáticas (Vieira et al., 2001; Visintin y Bernardello, 2005). Con respecto a la lámina foliar, la epidermis es simple y los estomas anomocíticos en los tres casos. Para estos caracteres Solereder (1908) y Metcalfe y Chalk (1979) señalan que son frecuentes en las Asteraceae, por lo que no pueden considerarse de valor diagnóstico a nivel de especie. Sin embargo, los apéndices epidérmicos sí ayudaron a distinguir a T. lucida y $T$. lunulata de T. micrantha, pues ésta fue la única que no presentó tricomas. El mesofilo en T. lucida y T. lunulata es bifacial, carácter común en esta familia (Metcalfe y Chalk, 1979; Milan et al., 2006). En contraste, en T. micrantha el mesofilo es de tipo equibilateral y el parénquima en empalizada, llega hasta los bordes internos del segmento. Esta diferencia no es evidente en todos los casos, ya que se llega a apreciar una gradación en la forma alargada de las células que limitan con la epidermis abaxial. El mesofilo equibilateral ha sido reportado por Delbón et al. (2007) para otros géneros de Asteraceae y parece estar asociado con cierto grado de xeromorfismo (Roth, 1984; Fahn, 1985) y como respuesta a los altos niveles de luz (Dickison, 2000). Además, la falta de compuestos nitrogenados, la iluminación intensa y un retardo en el flujo de agua, debido a la deficiencia hídrica, determinan el incremento del tejido en empalizada (Shields, 1950). Este tipo de mesofilo frecuentemente está asociado con hojas anfiestomáticas, como sucede en T. micrantha. Dentro del mesofilo se observaron canales secretores, reportados en diversas especies de Asteraceae (Milan et al., 2006; Delbón et al., 2007). Las diferencias más significativas se exhibieron a nivel de la vena media de las tres especies estudiadas. La presencia/ausencia y distribución de los tricomas y el número y organización de los cordones vasculares, constituyen un conjunto de caracteres que las separa. Por ejemplo, los tricomas en T. lucida se observaron en la superficie abaxial y en $T$. lunulata en la adaxial, mientras que en $T$. micrantha no existen. Con respecto a la vena media, las tres especies presentaron diferencialmente más de un cordón vascular con tamaños heterogéneos. Ello se observó en las tres especies estudiadas y de acuerdo con lo descrito por Metcalfe y Chalk (1979) es una característica presente en varios miembros de asteráceas. Para el género Flourencia, Delbón et al. (2007) registraron que cuando hay más de un haz los otros suelen ser de menor tamaño. También se diferenciaron en la vaina del haz de la vena media de la hoja de $T$. lucida y del segmento en T. lunulata y $T$. micrantha. En estas últimas especies la vaina está constituida por parénquima, mientras que en T. lucida se conforma de esclereidas.

A nivel del tallo una de las características diacríticas más evidentes son los tricomas. Tagetes lucida presenta tricomas escasos del tipo simple uniseriado con una célula terminal redondeada; en T. micrantha, los tricomas son de tipo glandular, abundantes en la base de sus ejes y escasos o ausentes hacia las partes más jóvenes del tallo, mientras que en $T$. lunulata su tallo es glabro. Es frecuente que la presenciaausencia y la forma de los apéndices epidérmicos aporten información en la clasificación de géneros, especies e híbridos interespecíficos (Metcalfe y Chalk, 1979).

El córtex también muestra diferencias marcadas entre las especies, pues Tagetes lucida y T. micrantha presentaron un aerénquima constituido por células unidas entre sí formando "cadenas" que se disponen laxamente. De igual manera este arreglo se reporta para el género Pluchea (Cambi et al., 2006). Los espacios intercelulares del aerénquima de las dos especies de Tagetes tienden a desaparecer en el córtex del tallo medio y superior, quedando reemplazados por espacios menos evidentes que se hallan entre el clorénquima y el colénquima. Contrariamente en $T$. lunulata las células parenquimáticas son de paredes delgadas y se organizan homogéneamente. En general, en las tres especies, el clorénquima es escaso y comienza a ser abundante en las partes medias y superiores del tallo. Las diferencias observadas en el esclerénquima asociado al floema, como el tamaño y el que se muestren discretos o no, se hicieron evidentes en las especies estudiadas. Fahn (1985) menciona que el esclerénquima es un tejido que sirve como soporte mecánico en los órganos donde se presenta; su disposición 
y abundancia puede servir de carácter diagnóstico, lo cual se confirmó para las especies estudiadas. Los tejidos de conducción primarios se organizan en haces vasculares del tipo colateral. El arreglo/acumulación del tejido vascular secundario muestra diferencias. Tagetes lucida acumula más floema en el área asociada a los paquetes de esclerénquima, en T. lunulata dicho tejido se desarrolla más hacia los ángulos y en $T$. micrantha la acumulación es uniforme alrededor del tallo. De la misma manera, el xilema secundario varía en acumulación; T. lunulata es la especie con mayor desarrollo xilemático, pero sus características anatómicas son semejantes en las tres especies.

Concluimos que los habitantes del municipio de Nicolás Romero, en el Estado de México, distinguen diferencialmente las tres especies de Tagetes estudiadas. Tienen conocimiento sobre la forma de prepararlas y administrarlas. Los usos registrados de las especies para contrarrestar problemas del aparato digestivo y/o como saborizantes de alimentos, son frecuentes en otras zonas del país, pero poco conocidos para padecimientos genito-urinarios. Tagetes lucida es la especie más conocida y la que registra más usos en Nicolás Romero. Los caracteres morfo-anatómicos de las hojas son los que contribuyen en mayor medida a la diferenciación entre las tres especies, siendo más evidentes los de la vena media y del mesofilo; el tejido cortical también aporta datos para su separación. La información etnobotánica y morfoanatómica de las especies estudiadas se complementa con el estudio fitoquímico realizado por Downum y Towers (1983), en donde se reportan diferencias de T. lunulata con T. lucida y T. micrantha. La información obtenida aporta datos útiles para la incorporación de las tres especies de Tagetes a la Farmacopea Herbolaria de los Estados Unidos Mexicanos.

\section{Agradecimientos}

Las autoras agradecen a los revisores anónimos por sus valiosas sugerencias realizadas al trabajo y a María Patricia Jácquez Ríos, por la revisión de la primera versión del manuscrito.

\section{Literatura citada}

Aguilar A., Camacho J.R., Chino S., Jácquez P. y López M.E. 1994. Herbario Medicinal del Instituto Mexicano del Seguro Social. Información Etnobotánica. Instituto Mexicano del Seguro Social, México, D.F.

Aguilar-Rodríguez S. 1998. Técnicas de laboratorio para el estudio de las embriofitas. En: Tejero-Díez J.D. y Granillo-Velázquez M.P. Eds. Plantae. Introducción al Estudio de las Plantas con Embrión, pp. 247-272, Universidad Nacional Autónoma de México, México, D.F.

Aguilar-Rodríguez S., Terrazas T. y López-Mata L. 2006. Anatomical wood variation of Buddleja cordata (Buddlejaceae) along its natural range in Mexico. Trees: Structure and Function 20:253-261.
Alonso-Castro A.J., Villarreal M.L., Salazar-Olivo L.A., GomezSanchez M., Dominguez F. y Garcia-Carranca A. 2011. Mexican medicinal plants used for cancer treatment: pharmacological, phytochemical and ethnobotanical studies. Journal of Ethnopharmacology 133:945- 972.

Argueta-Villamar A., Cano-Asseleih L.M. y Rodarte M.E. 1994. Atlas de las Plantas de la Medicina Tradicional Mexicana. Instituto Nacional Indigenista, México, D.F.

Bicchi C., Fresia M., Rubiolo P., Monti D., Chlodwig F. y Goehler I. 1996. Constituents of Tagetes lucida Cav. ssp. lucida essential oil. Flavour and Fragance Journal 12:47-52.

Caballero J. 1990. El uso de la diversidad vegetal en México. Tendencias y perspectivas futuras. En: Leff E. Coord. Medio Ambiente y Desarrollo en México volumen primero, pp. 257-298, Centro de Investigaciones Interdisciplinarias en Humanidades/ Universidad Nacional Autónoma de México, México, D.F.

Cáceres A., Jáuregui E., López Br. y Logemann H. 1992. Actividad Antifúngica de Plantas de Uso Medicinal en Guatemala. Cuadernos DIGI 7-92, Dirección General de Investigación/Unidad de San Carlos de Guatemala, Guatemala.

Cambar P., Tabora E., Andonie H.I., Alger J., Figueroa R., Martínez E. y Martínez J. 2000. Estudio preliminar de los efectos farmacológicos de Tagetes lucida (pericón). Revista Médica Hondureña 52:142-147.

Cambi V., Bucciarelli A., Flemmer A. y Hansen P. 2006. Morfoanatomía de Pulchea sagittalis (Asteraceae), especie nativa de interés medicinal. Acta Farmacéutica Bonaerense 25:43-49.

Campos-Navarro R. 1997. La Antropología Médica en México. Instituto Mora/Universidad Autónoma Metropolitana, México, D.F.

Ciccio J.F. 2004. A source of almost pure methyl chavicol: volatile oil from the aerial parts of Tagetes lucida (Asteraceae) cultivated in Costa Rica. Revista de Biología Tropical 52:853-857.

Cotton C.M. 1996. Ethnobotany: Principles and Applications. John Wiley \& Sons, Chichester.

CPFEUM [Comisión Permanente de la Farmacopea de los Estados Unidos Mexicanos]. 2001. Farmacopea Herbolaria de los Estados Unidos Mexicanos. Secretaria de Salud/Comisión permanente de la Farmacopea de los Estados Unidos Mexicanos, México, D.F.

de la Cruz M. y Badiano J. 1991. Libellus de Medicinalibus Indorum Herbis, manuscrito azteca de 1552, Volume 1. Fondo de Cultura Económica, México, D.F.

Delbón N., Cosa M.T. y Dottori N. 2007. Anatomía de órganos vegetativos en Flourencia campestris y F. oolepis (Asteraceae), con especial referencia en las estructuras secretoras. Arnaldoa 14:61-70.

Dickison W.C. 2000. Integrative Plant Anatomy. Academic Press, San Diego.

Downum K.R. y Towers G.H.N. 1983. Analysis of thiophenes in the Tageteae (Asteraceae) by Hplc. Journal of Natural Products 46:98-103.

Fahn A. 1985. Anatomía Vegetal. Pirámide, S.A., Madrid.

Font Quer P.1982. Diccionario de Botánica. Labor, S.A. de C.V, Barcelona.

Foster G.M. 1988. The validating role of humoral theory in traditional Spanish-American therapeutics. American Ethnologist 15:120-135.

García E. 1973. Modificaciones al Sistema de Clasificación Climática de Köppen (para adaptarlo a las condiciones de la República Mexicana). Universidad Nacional Autónoma de México/ 
Instituto de Geografía, México, D.F.

Hernández T., Canales M., Flores C., García A.M., Durán A. y Ávila J.G. 2006. Antimicrobial activity of Tagetes lucida. Pharmaceutical Biology 44:19-22.

Hernández-X E. 1979. El concepto de etnobotánica. En: Barrera A. Ed. La Etnobotánica: Tres Puntos de Vista y una Perspectiva, pp. 13-18, Instituto de Investigaciones Sobre Recursos Bióticos A.C., Xalapa.

Howard P.L. 2003. Women and Plant. Gender Relations in Biodiversity Management, and Conservation. Zed Books, Londres.

IAWA Committee. 1989. IAWA list of microscopic features for hardwood identification. International Association of Wood Anatomists Bulletin New Series 10:219-232.

Lira R., Casas A., Rosas-López R., Paredes-Flores M., PérezNegrón E., Solís L., Torres I. y Dávila P. 2009. Traditional knowledge and useful plant richness in the Tehuacán-Cuicatlán Valley, Mexico. Economic Botany 63:271-287.

Lot A. y Chiang F. 1986. Manual de Herbario: Administración y Manejo de Colecciones, Técnicas de Recolección y Preparación de Ejemplares Botánicos. Consejo Nacional de la Flora de México, México, D.F.

Martínez-Alfaro M.A. 1976. Posible metodología a seguir en el estudio de las plantas medicinales. En: Viesca-Treviño C. Ed. Estudios sobre Etnobotánica y Antropología Médica, pp. 75-83, Instituto Mexicano para el Estudio de las Plantas Medicinales, A. C., México, D.F.

Metcalfe C.R. y Chalk L. 1979. Anatomy of the Dycotiledons, Volume I: Systematic Anatomy of Leaf and Stem with a Brief History of the Subject. Oxford University Press, Oxford.

Milan P., Hissae A.H. y Appezzato-da Glória B. 2006. Comparative leaf morphology and anatomy of three Asteraceae species. Brazilian Archives of Biology and Technology 49:135-144.

Moles A.T., Warton D.I., Warman L., Swenson N.G., Laffan S.W., Zanne A.E., Pitman A., Hemmings F.A. y Leishman M.R. 2009. Global patterns in plant height. Journal of Ecology 97:923-932.

Nates-Cruz B., Cerón P. y Hernández E.1996. Las Plantas y el Territorio. Clasificaciones, Usos y Concepciones en los Andes Colombianos. Hombre y Ambiente No. 37 Abya-Yala/Corporación Ambiental Madremonte, Quito.

Nikon Corporation. 1991-2006. NIS- Elements, versión 2.33

Ogunwande I.A. y Olawore N.O. 2006. The essential oil from the leaves and flowers of African Marigold "Tagetes erecta L.". The Journal of Essential Oil Research 18:366-368.

Osuna-Torres L., Tapia-Pérez M.E. y Aguilar-Contreras A. 2005. Plantas Medicinales de la Medicina Tradicional Mexicana para Tratar Afecciones Gastrointestinales: Estudio Etnobotánico, Fitoquímico y Farmacológico. Universidad de Barcelona, Barcelona.

Roth I. 1984. Stratification of Tropical Forests as Seen in Leaf Structure. Dr. W. Junk, La Haya.

Russin W.A., Uchytil T.F., Feistner G. y Durbin R.D. 1988. Developmental changes in content of folia secretory cavities of Tagetes erecta (Asteraceae). American Journal of Botany 75:1787-1793.

Ruzin S.E. 1999. Plant Microscopy and Microtechnique. Oxford
University Press, Nueva York.

Rzedowski J. 1978. Vegetación de México. Limusa, México, D.F.

Rzedowski J. 2001. Compositae. En: Rzedowski G.C. de, y Rzedowski J. Eds. Flora Fanerogámica del Valle de México, pp. 764-975, Instituto de Ecología y Comisión Nacional para el Conocimiento y Uso de la Biodiversidad, Pátzcuaro.

Sandoval-Z E. 2005. Técnicas Aplicadas al Estudio de la Anatomía Vegetal. Instituto de Biología. Universidad Nacional Autónoma de México, México, D.F.

Serrato-Cruz M.A. 2003. Estado actual del conocimiento sobre Tagetes como ornamental. En: Mejía-Muñoz J.M. y Espinosa-Flores A. Eds. Plantas Nativas de México con Potencial Ornamental. Análisis y Perspectiva, pp. 189-198, Universidad Autónoma Chapingo, Chapingo.

Serrato-Cruz M.A. y Barajas-Pérez J.S. 2006. Poblaciones silvestres de Tagetes filifolia Lag. en el centro y sur de México. Revista Fitotecnia Mexicana 29:7-12.

Serrato-Cruz M.A., Díaz-Cedillo F. y Barajas-Pérez J.S. 2008. Composición del aceite esencial en germoplasma de Tagetes filifolia Lag. de la región centro-sur de México. Agrociencia 42:277-285.

Shields L.M. 1950. Leaf xeromorphy as related to physiological and structural influences. Botanical Review 16:399-447.

Simon P.M., Katinas L. y Arambarri A.M. 2002. Secretory structures in Tagetes minuta (Asteraceae, Heleniae). Boletín de la Sociedad Botánica de Argentina 37:181-191.

Solereder H. 1908. Systematic Anatomy of the Dicotyledons: A Handbook for Laboratories of Pure and Applied Botany. Clarendon Press, Oxford.

Strother J.L. 1977. Tagetes-systematic review. En: Heywood V.H., Harborne J.B. y Turner B.L. Eds. The Biology and Chemistry of the Compositae, pp. 769-783, Academic Press, Londres.

Vieira R.C., Delprete P.G, Leitão G.G. y Leitão S.G. 2001. Anatomical and chemical analyses of leaf secretory cavities of Rustia formosa (Rubiaceae). American Journal of Botany 88:2151-2156.

Villarreal Q.J.Á. 2003. Familia Compositae, Tribu Tageteae. Fascículo 113 de Flora del Bajío y de Regiones Adyacentes. Instituto de Ecología A.C., Pátzcuaro.

Villaseñor J.L., Maeda P., Colín-López J.J. y Ortíz E. 2005. Estimación de la riqueza de especies de Asteraceae mediante extrapolación a partir de datos de presencia-ausencia. Boletín de la Sociedad Botánica de México 76:5-18.

Villavicencio-Nieto M.A., Pérez-Escandón B.E. y Gordillo-Martínez A.J. 2010. Plantas tradicionalmente usadas como plaguicidas en el estado de Hidalgo, México. Polibotánica 30:193-238.

Visintin A.M. y Bernardello G. 2005. Morfología y anatomía floral de Tagetes minuta L. (Asteraceae). Arnaldoa 12:8-15.

Zolla C., Del Bosque S., Tascon A. y Mellado V. 1998. Medicina Tradicional y Enfermedad. Centro Interamericano de Estudios de Seguridad Social/Instituto Mexicano del Seguro Social, México, D.F.

Zolla C. y Zolla-Márquez E. 2004. Los Pueblos Indígenas de México: 100 preguntas. Universidad Nacional Autónoma de México, México, D.F. 